Lina Martin Hita

Isabel Casanovas Moreno-

Torre

Jaime Borrego Jiménez

Carla Foronda García-

Hidalgo

Vicente Guillot Suay

Jose María Navarro Mari

\title{
Listeria monocytogenes, a rare cause of endophthalmitis
}

Department of Microbiology. Hospital Virgen de las Nieves, Granada. Instituto de Investigación Biosanitaria, Granada.

\section{Article history}

Received: 29 November 2019; Revision Requested: 3 March 2020; Revision Received: 29 March 2020; Accepted: 3 April 2020;

Published: 13 May 2020

\section{Sir,}

Listeria monocytogenes is a ubiquitous Gram-positive rod that is found in food and is responsible for gastrointestinal infections due to the consumption of unpasteurized dairy products or contaminated meat and vegetables. Rarely, it produces invasive disease that primarily affects immunosuppressed patients, chronic diseases, intravenous drug use, malignancies, the elderly and pregnant women; mainly causing bacteremia and meningitis [1-3]. L. monocytogenes is a rare and serious cause of endophthalmitis, which can lead to vision loss [3]. The first case reported in the literature of endophthalmitis due to L. monocytogenes was in 1967 by Goodner and Okumoto [4], since then have been published 25 cases. Endophthalmitis of endogenous cause, less frequent than the exogenous one, is due to blood-borne seeding from a place far from the eye and is related to the presence of predisposing factors, such as cirrhosis or haemochromatosis [5].

We present a case report of endogenous endophthalmitis due to $L$. monocytogenes in a cirrhotic patient. A 55-year-old patient presented a painful red eye and visual disturbance in the right eye for 1 month. His personal history includes liver cirrhosis due to Hepatitis C virus (HCV) and ethanol, having started 2 weeks before the antiviral treatment for HCV. The temporal relationship between the initiation of antiviral treatment and the clinical uveitis made the physicians suspect a causal relationship (there are cases described), so the treatment was suspended. Several serological tests were requested in the aqueous humor for screening of acute infectious pathology in an immunosuppressed patient. Valganciclovir oral

Correspondence:

Isabel Casanovas Moreno-Torres

Department of Microbiology. Hospital Virgen de las Nieves. Granada. Avda. de las Fuerzas Armadas 2, 18014.

E-mail: isabelcmt@hotmail.com plus ophthalmological treatment was empirically initiated, without clear improvement. In view of the poor evolution of panuveitis, a vitrectomy was performed and sent for culture and virus study to microbiology.

The sample was cultured on aerobic and anaerobic blood agar (Beckton Dikinson Columbia Agar 5\%), chocolate agar and thioglycolate. The study of the virus in the vitreous humor (simple herpes virus, virus varicella zoster and cytomegalovirus) was negative. In Gram stain, Gram-positive bacilli were observed and after 18 hours of incubation, gram-positive bacilli grew in pure culture on both, blood and chocolate agar, catalase positive and oxidase negative. They were identified by mass spectrometry (MALDI-TOF MS, Bruker Biotyper) as $\mathrm{L}$. monocytogenes with a 2,25 score. The minimun inhibitory concentration (MIC) to the different antibiotics was performed by the MIC test method on Mueller Hinton agar with $5 \%$ sheep blood and incubated at $37^{\circ} \mathrm{C} 24 \mathrm{~h}$. The MIC values were interpreted following the EUCAST cutoffs for Listeria. $L$. monocytogenes was susceptible to ampicillin ( $0.5 \mathrm{mg} / \mathrm{L})$, erythromycin $(0.25 \mathrm{mg} / \mathrm{L})$ and meropenem $(0.19 \mathrm{mg} / \mathrm{L})$ and resistant to trimethoprim/sulfamethoxazole $(0.19 \mathrm{mg} / \mathrm{L})$. Treatment was started intravenously with ampicillin for 3 weeks, associating gentamicin for 2 weeks. At the ophthalmologic level, the treatment was changed to intravitreal ofloxacin (every 6 hours for 10 days). The sample was sent to the National Center of Microbiology for its characterization and the result was L. monocytogenes group $4(\mathrm{IVb})$. Blood cultures were negative and the origin of the infection could not be established, although it could be ruled out that it was due to an exogenous cause due to the absence of previous treatment or eye surgery.

Most of patients who present endophthalmitis due to $L$. monocytogenes have poor visual acuity results even with adequate antibiotic treatment, especially due to a delay in the diagnosis since it is not a frequent pathology [1]. Because of 
this, a diagnosis and early treatment of endophthalmitis due to L. monocytogenes is crucial, and should be considered in the differential diagnosis of immunosuppressed patients with anterior uveitis and high intraocular pressure [6]. In our case, the diagnosis was delayed one month after presenting the symptoms, which explains the non-recovery of visual acuity, even with the appropriate antibiotic treatment. In endogenous endophthalmitis it is common not to find the source of the infection, despite taking samples for its search, as it happened in our case.

Ampicillin is the treatment of choice, and it was the antibiotic used in this patient [7]. In summary, it is crucial to highlight the importance of taking samples for microbiological culture for the diagnosis of endophthalmitis due to L. monocytogenes, since they are the key to the diagnosis and early treatment of this pathology so difficult to diagnose due to its rare but serious presentation.

\section{FUNDING}

None to declare

\section{CONFLICT OF INTEREST}

The authors declare that they have no conflicts of interest

\section{REFERENCES}

1. Weber Adam C, Levison Ashleigh L, Srivastava Sunil K, Lowder Careen $\mathrm{Y}$. A case of Listeria monocytogenes endophthalmitis with recurrent inflammation and novel management. J Ophthalmic Inflamm Infect 2015; 5:28. doi: 10.1186/s12348-015-0058-8.

2. Gaskell K, Williams G, Grant K, Lightman S. Listeria monocytogenes: a rare cause of endophthalmitis, a case report. IDCases 2017; 8: 45-46. doi: 10.1016/j.idcr.2017.03.004

3. Betriu C, Fuentemilla S, Méndez R, Picazo J., Garcia-Sánchez J. Endophthalmitis caused by Listeria monocytogenes. J Clin Microbiol. 2001;39(7):2742-4. doi: 10.1128/JCM.39.7.2742-2744.2001.

4. Goodner EK, Okumoto M. Intraocular listeriosis. Am J Ophthalmol. 1967; 64:682-686.

5. Pérez-Carro G., García-Alonso A., Corte-Torres G., Viejo G. Endoftalmitis por Listeria monocytogenes: A propósito de un caso. Arch Soc Canar Oftal. 2011; 22: 62-65.

6. Bajor A, Luhr A, Brockmann D, Suerbaum S, Framme C, Sedlacek L. Listeria monocytogenes endophthalmitis-case report and review of risk factors and treatment outcomes. BMC Infectious Diseases 2016, 16: 332. doi: 10.1186/s12879-016-1680-2.

7. Shoughy SS, Tabbara KF. Listeria monocytogenes endophthalmitis following keratoconjunctivitis. Clin Ophthalmol. 2014; 8:301-4. doi: 10.2147/OPTH.S55710. 